\title{
Some fixed point theorems in metric spaces
}

by

\author{
Shigeru Itoh (Tokyo)
}

Abstract. We will consider various fixed point theorems in metric spaces with some structure. First, we will give theorems for multivalued mappings. Then applying these results, we will prove common fixed point theorems for singlevalued mappings and multivalued mappings.

1. Introduction. Takahashi [11] considered metric spaces with some convex structure and proved fixed point theorems for nonexpansive mappings. Machado [8] further studied convex structures in metric spaces including the one initiated by Takahashi (which Machado called $W$-convexity) and gave various fixed point theorems. Takahashi or Machado's main results were generalizations of known fixed point theorems for amenable semigroups of nonexpansive mappings on compact convex subsets or for commutative families of nonexpansive mappings on weakly compact convex subsets of Banach spaces respectively (cf. Takahashi [10], Belluce-Kirk [1]).

In this paper we will prove fixed point theorems for multivalued mappings and common fixed point theorems for singlevalued and multivalued mappings in $W$-starshaped and $W$-convex metric spaces.

The author wishes to express his thanks to Professors H. Umegaki and W. Takahashi for many suggestions and encouragements in preparing this paper.

2. Preliminaries. Let $X$ be a complete metric space with the metric $d$. For any $A \subset X$ and $c>0$, we denote $d(x, A)=\inf \{d(x, y): y \in A\}$ and

$$
V(A, c)=\{y \in X: d(y, A)<c\} .
$$

Let $2^{X}$ be the family of all subsets of $X, \mathrm{CB}(X)$ all nonempty bounded closed subsets of $X, K(X)$ all nonempty compact subsets of $X$ respectively. Let $D$ be the Hausdorff matric on $\mathrm{CB}(X)$ induced by $d$, that is, if $A, B \in \mathrm{CB}(X)$, then

$$
D(A, B)=\max \left\{\sup _{x \in A} d(x, B), \sup _{y \in B} d(y, A)\right\} .
$$

DeFinition 1. For any nonempty bounded subset $A$ of $X$, we define $\gamma(A)=\inf \{c>0: A$ can be covered by a finite number of subsets of $X$ whose 
diameters are less than or equal to $c\} . \gamma(A)$ is called the (set-) measure of noncompactness of $A$.

The idea of measure of noncompactness is due to Kuratowski (cf. [3]).

Let $f$ be a mapping of $X$ into $X . f$ is said to be $k$-Lipschitz $(k \geqslant 0)$ if for every $x, y \in X, d(f x, f y) \leqslant k d(x, y)$. $k$-Lipschitz mappings are called $k$-contraction or nonexpansive if $0 \leqslant k<1$ or $k=1$ respectively. $f$ is said to be quasi-nonexpansive if $F(f)=\{x \in X: f x=x\}$ is nonempty and for each $x \in X, u \in F(f), d(f x, u)$ $\leqslant d(x, u)$. $f$ is said to be asymptotically regular if for any $x \in X, d\left(f^{n} x, f^{n+1} x\right) \rightarrow 0$ as $n \rightarrow \infty$. $f$ is said to be condensing if $f$ is continuous and for each $A \subset X$ with $\gamma(A)>0$, $\gamma(f(A))<\gamma(A)$.

Let $T$ be a mapping of $X$ into $\mathrm{CB}(X) . T$ is called $k$-Lipschitz $(k \geqslant 0)$ if for any $x, y \in X, D(T x, T y) \leqslant k d(x, y)$. When $0 \leqslant k<1$ or $k=1, k$-Lipschitz mappings are called $k$-contraction or nonexpansive respectively. $T$ is called upper semicontinuous if for every closed subset $C$ of $X, T^{-1}(C)=\{x \in X: C \cap T x \neq \varnothing\}$ is a closed subset of $X$. When $T$ is a $k$-Lipschitz mapping of $X$ into $K(X)$, then $T$ is upper semicontinuous. $T$ is called condensing if $T$ is upper semicontinuous and for any $A \subset X$ such that $\gamma(A)>0, \gamma(T(A))<\gamma(A)$, where $T(A)=\bigcup\{T x: x \in A\} . T$ is called asymptotically regular if for each $x_{0} \in X$, there exists a sequence $\left\{x_{n}\right\} \subset X$ such that $x_{n+1} \in T x_{n}(n=0,1,2, \ldots)$ and $d\left(x_{n}, x_{n+1}\right) \rightarrow 0$ as $n \rightarrow \infty$.

Definition 2. A metric space $X$ is said to be $W$-starshaped if there exist an $x_{0} \in X$ and a mapping $W: X \times\left\{x_{0}\right\} \times[0,1] \rightarrow X$ such that for each $x, y \in X, a \in[0,1]$,

$$
d\left(x, W\left(y, x_{0}, a\right)\right) \leqslant a d(x, y)+(1-a) d\left(x, x_{0}\right) .
$$

A metric space $X$ is said to be $W$-convex if there exists a mapping $W: X \times X \times[0,1] \rightarrow X$ such that for every $x, y, z \in X, a \in[0,1]$,

$$
d(x, W(y, z, a)) \leqslant a d(x, y)+(1-a) d(x, z) .
$$

A subset $K$ of a $W$-convex metric space is said to be convex if for any $x, y \in K$, $a \in[0,1], W(x, y, a) \in K$.

Defintion 3. A $W$-starshaped metric space $X$ is said to satisfy condition (I) if for any $x, y \in X, a \in[0,1]$,

$$
d\left(W\left(x, x_{0}, a\right), W\left(y, x_{0}, a\right)\right) \leqslant a d(x, y) .
$$

A $W$-convex metric space $X$ is said to satisfy condition (II) if for every $x, y, z \in X$, $a \in[0,1]$,

$$
d(W(x, z, a), W(y, z, a)) \leqslant a d(x, y) .
$$

A $W$-convex metric space $X$ is said to satisfy condition (III) if for each $x, y, u, v \in X$, $a \in[0,1]$

$$
d(W(x, y, a), W(u, v, a)) \leqslant a d(x, u)+(1-a) d(y, v) .
$$

$W$-starshaped metric spaces are generalizations of starshaped subsets of Banach spaces, where a subset $K$ of a Banach space is starshaped if there exists an $x_{0}$ in $K$ such that for each $x \in K, a \in[0,1], a x+(1-a) x_{0} \in K$. It is obvious that $W$-convex metric spaces are $W$-starshaped, and $W$-convex metric spaces satisfying condition (III) satisfy condition (II). When $X$ is $W$-convex, we denote CK $(X)$ the family of all nonempty compact convex subsets of $X$.

Let $X$ be a Hausdorff topological space, $C(X)$ the Banach space of all bounded real valued continuous functions on $X$ with sup norm. Let $A$ be a closed subspace of $C(X)$ containing $1_{X}$ (the constant 1 function on $\left.X\right), A^{*}$ its dual space. $m \in A^{*}$ is called a mean on $A$ if $\|m\|=m\left(1_{X}\right)=1$.

Let $G$ be a semitopological semigroup, that is, $G$ is a semigroup and a Hausdorff topological space such that the semigroup operation of $G$ is separately continuous. $G$ is said to act on a topological space $X$ if there exists a mapping $G \times X \rightarrow X$ such that $(s t) x=s(t x)$ for every $s, t \in G, x \in X$, where $t x$ is the image of $(t, x)$. The action is said to be separately continuous if the mapping $G \times X \rightarrow X$ is separately continuous. The action of $G$ on $X$ is said to be equicontinuous if the family of mappings $\{x \rightarrow s x(x \in X): s \in G\}$ is equicontinuous. In particular, if $X$ is a metric space and the mappings $\{x \rightarrow s x(x \in X): s \in G\}$ are nonexpansive, then the action is called nonexpansive.

For any $s \in G$, define operators $l_{s}, r_{s}: C(G) \rightarrow C(G)$ by $\left(l_{s} h\right)(t)=h(s t)$ and $\left(r_{s} h\right)(t)=h(t s)(h \in C(G), t \in G)$. A subspace $A$ of $C(G)$ is called translation invariant if for every $s \in G, l_{s}(A), r_{s}(A) \subset A$. Let $A$ be a translation invariant subspace containing $1_{G}$, then a mean $m$ on $A$ is called a left invariant mean if $m\left(l_{s} h\right)=m(h)$ for any' $h \in C(G), s \in G . h \in C(G)$ is called almost periodic if $\mathrm{OL}(h)=\left\{l_{s} h: s \in G\right\}$ is $h \in C(G), s \in G . h \in C(G)$ is called almost periodic invariant closed subalgebra of $C(G)$ containing $1_{G}$. It is known that $h \in \operatorname{AP}(G)$ if and only if $\operatorname{OR}(h)=\left\{r_{s} h: s \in G\right\}$ is precompact. If $G$ is left reversible, then $\operatorname{AP}(G)$ has a left invariant mean (cf. [4], [7]).

3. Fixed point theorems for multivalued mappings. We will extend a result in [3] to the case of multivalued mappings in the follwing way.

Proposition 1. Let $X$ be a complete metric space, $T$ a condensing mapping of $X$ into $\mathrm{CB}(X)$. Suppose there is a nonempty bounted subset $K$ of $X$ such that $T(K)$ is bounded and $\inf \{d(x, T(x)): x \in K\}=0$. Then there exists a fixed point $z \in \mathrm{cl}(K)$, the closure of $K$, of $T$, i.e., $z \in T z$.

Proof. Choose a sequence $\left\{x_{n}\right\} \subset K$ such that $d\left(x_{n}, T\left(x_{n}\right)\right) \leqslant 2^{-n}(n=1,2, \ldots)$ For each $n$, there exists a $y_{n} \in T\left(x_{n}\right)$ for which

$$
d\left(x_{n}, y_{n}\right) \leqslant d\left(x_{n}, T\left(x_{n}\right)\right)+2^{-n} \leqslant 2^{-n+1} .
$$

For any $c>0$, take a $N=N(c)$ such that $2^{-N+1} \leqslant c$, then for each $n>N, d\left(x_{n}, y_{n}\right)<c$. Denote $A=\left\{x_{n}\right\}, B=\left\{y_{n}\right\}$ and $C=\left\{x_{1}, x_{2}, \ldots, x_{N}\right\}$, then $V(B, c) \supset A \backslash C$ and $\gamma(A)=\gamma(A \backslash C) \leqslant \gamma(V(B, c)) \leqslant \gamma(B)+2 c$.

As $c$ is arbitrary, we obtain

$$
\gamma(A) \leqslant \gamma(B) \leqslant \gamma(T(A)) .
$$


This implies $A$ is precompact, since $T$ is condensing. Thus, there exists a subsequence $\left\{x_{m}\right\}$ of $\left\{x_{n}\right\}$ such that $\left\{x_{m}\right\}$ converges to some $z \in \operatorname{cl}(K)$. Then $\left\{y_{m}\right\}$ converges to $z$ and, since $T$ is upper semicontinuous, we have $z \in T(z)$. Q.E.D.

Now we will give a fixed point theorem in $W$-starshaped metric spaces.

THEOREM 1. Let $X$ be a bounded complete $W$-starshaped metric space satisfying condition (I). Let $T$ be a nonexpansive condensing mapping of $X$ into $K(X)$, then there exists a fixed point of $T$ in $X$.

Proof. There are an $x_{0} \in X$ and a mapping $W: X \times\left\{x_{0}\right\} \times[0,1] \rightarrow X$. For any $k \in(0,1)$, define a mapping $T_{k}: X \rightarrow 2^{X}$ by $T_{k}(x)=\left\{W\left(y, x_{0}, k\right): y \in T(x)\right\}$ $(x \in X)$, then for any $x \in X, T_{k}(x)$ is nonempty compact and $T_{k}$ is $k$-contraction, since $X$ satisfies condition (I), Thus, by [9] there are a fixed point $x_{k} \in X$ of $T_{k}$ and a $y_{k} \in T\left(x_{k}\right)$ such that $x_{k}=W\left(y_{k}, x_{0}, k\right)$. It follows that

$$
d\left(x_{k}, T\left(x_{k}\right)\right) \leqslant d\left(x_{k}, y_{k}\right)=d\left(W\left(y_{k}, x_{0}, k\right), y_{k}\right) \leqslant(1-k) d\left(x_{0}, y_{k}\right) .
$$

Since $X$ is bounded, $d\left(x_{k}, T\left(x_{k}\right)\right) \rightarrow 0$ as $k \rightarrow 1$. By Proposition 1, there exists a fixed point of $T$ in $X$. Q.E.D.

When $X$ is compact, we can easily derive the following

COROLlary 1. Let $X$ be a compact $W$-starshaped metric space satisfying condition (I). Let $T$ be a nonexpansive mapping of $X$ into $K(X)$, then there is a fixed point of $T$ in $X$.

For asymptotically regular mappings, the following theorem holds.

THEOREM 2. Let $X$ be a bounded complete metric space, $T$ an asymptotically regular, condensing mapping of $X$ into $\mathrm{CB}(X)$. Then there exists a fixed point of $T$ in $X$.

Proof. Fix an element $x_{0} \in X$ arbitrarily. Since $T$ is asymptotically regular, there is a sequence $\left\{x_{n}\right\} \subset X$ such that $x_{n+1} \in T x_{n}(n=0,1, \ldots)$ and $d\left(x_{n}, x_{n+1}\right) \rightarrow 0$ as $n \rightarrow \infty$. Then we have $d\left(x_{n}, T x_{n}\right) \rightarrow 0$ as $n \rightarrow \infty$. By Proposition 1, there is a fixed point of $T$ in $X$. Q.E.D.

4. Common fixed point theorems. We will state a fundamental result which is essentially contained in [7, p. 74]. For the sake of completeness we will give its proof.

Proposition 2. Let $X$ be a compact Hausdorff space, $G$ a semitopological semigroup acting on $X$. Suppose the action of $G$ on $X$ is separately continuous and equicontinuous. If $\mathrm{AP}(G)$ has a left invariant mean, then there exists a nonempty compact subset $M$ of $X$ such that $s(M)=M$ for any $s \in G$.

Proof. By Zorn's lemma, there exists a minimal nonempty closed subset $M$ of $X$ which is $G$-invariant, i.e., for any $s \in G, s(M) \subset M$. Define a mapping $Q: X \times C(M) \rightarrow C(G)$ by $Q(x, h)(s)=h(s x)(x \in X, h \in C(M), s \in G)$, then by [7] the range of $Q$ is contained in $\operatorname{AP}(G)$. Let $m$ be a left invariant mean on $\operatorname{AP}(G)$ and fix a point $y \in M$. Define a mapping $p: C(M) \rightarrow R$ by $p(h)=m(Q(y, h))$ $(h \in C(M))$, then $p$ is a mean on $C(M)$ and for every $s \in G, p\left(L_{s} h\right)=p(h)$, where $L_{s}$ is the operator on $C(M)$ defined by $\left(L_{s} h\right)(x)=h(s x)(x \in M)$. Thus, by Riesz's theorem there is a probability measure $P$ on $M$ which is invariant under each $s \in G$. Put $M_{0}=\bigcap\{F \subset M: F$ is closed and $P(F)=1\}$, then $M_{0}$ is nonempty closed and $G$-invariant. Since $M$ is minimal, $M=M_{0}$. For each $s \in G$,

$$
P(s(M))=P\left(s^{-1} s(M)\right)=P(M)=1,
$$

hence $M \supset s(M) \supset M_{0}=M$ and $s(M)=M$. Q.E.D.

The following theorem is an extension of a result in [8, p. 50]. The method of the proof is a slight modification of those in $[4,8]$.

THEOREM 3. Let $X$ be a bounded W-convex metric space satisfying condition (II), $K$ a compact subset of $X, G$ a semitopological semigroup actioning on $X$. Suppose the action of $G$ on $X$ is separately continuous and nonexpansive. If $\operatorname{AP}(G)$ has $a$ left invariant mean and if there is an element $t \in G$ such that $t$ commutes with every $s \in G$ and for any $x \in X, \operatorname{cl}\left\{t^{n} x: n=1,2, \ldots\right\}$ contains a point of $K$, then there exists a common fixed point $z \in K$ of $G$, i.e., $s z=z$ for all $s \in G$.

Proof. There exists a minimal nonempty closed convex $G$-invariant subset $Y$ of $X$, since each nonempty closed convex $G$-invariant subset of $X$ intersects with $K$. As in the proof of Theorem 1 , $\inf \{d(x, t x): x \in Y\}=0$ because $X$ satisfies condition (II). For each $x \in X, \operatorname{cl}\left\{t^{n} x: n=1,2, \ldots\right\}$ contains a point of $K$, thus $\inf \{d(x, t x): x \in Y\}=\min \{d(x, t x): x \in Y \cap K\}$ and there is a fixed point of $t$ in $\dot{Y} \cap K$. Let $F$ be the fixed point set of $t$ in $Y \cap K$, then $F$ is $G$-invariant and compact. By Proposition 2 there exists a nonempty compact subset $M$ of $F$ such that for any $s \in G, s(M)=M$. If $M$ contains more than one point, then by [11, Proposition 5] there is a $u \in Y$ for which $\sup \{d(u, x): x \in M\}=r<\operatorname{diam}(M)$ (the diameter of $M$ ). Put $Y_{0}=\{y \in Y: d(x, y) \leqslant r$ for all $x \in M\}$, then $Y_{0}$ is a nonempty closed convex $G$-invariant proper subset of $Y$. This contradicts the minimality of $Y$. Therefore $M$ consists of a single point which is a fixed point of $G$. Q.E.D.

When $X$ itself is compact, we have the following

THEOREM 4. Let $X$ be a compact $W$-convex metric space, $G$ a semitopological semigroup acting on $X$. Suppose the action of $G$ on $X$ is separately continuous and nonexpansive. If $\operatorname{AP}(G)$ has a left invariant mean, then there is a common fixed point of $G$ in $X$.

Proof. Let $K$ be any $G$-invariant compact convex subset of $X$, then by Proposition 2 there exists a nonempty compact subset $M$ of $K$ such that for each $s \in G$, $s(M)=M$. Hence, by [11, Theorem 3] $G$ has a common fixed point in $X$. Q.E.D.

Now we will give an interesting result which will be used to prove a common fixed point theorem for a semigroup of nonexpansive mappings and a multivalued nonexpansive mapping. A mapping $r$ of a set $X$ onto a subset of $X$ is called a retraction if $r^{2}=r$.

Proposition 3, Let $X$ be a compact $W$-convex metric space satisfying condition (III), $G$ a semitopological semigroup acting on $X$. If the action of $G$ on $X$ is separately continuous, nonexpansive and if $\operatorname{AP}(G)$ has a left invariant mean, then 3 - Fundamenta Mathematicae CII 
there exists a nonexpansive retraction $r$ of $X$ onto $F(G)$ (the fixed points of $G$ in $X$ ) such that every $G$-invariant closed convex subset of $X$ is $r$-invariant.

Proof. $F(G)$ is nonempty compact by Theorem 4. Let $S=\{f: X \rightarrow X: f$ is nonexpansive with $F(f) \supset F(G)$ and every $G$-invariant closed convex subset of $X$ is $f$-invariant $\}$, then $S$ contains the mappings $\{x \rightarrow s x(x \in X): s \in G\}$. Fix an element $u \in F(G)$. For any $x \in X$, denote $H x=\{y \in X: d(y, u) \leqslant d(x, u)\}$, then $H x \supset G x$ $=\{s x: s \in G\}$ and $H x$ is compact convex. Hence, the product topological space $H=\prod H x$ is compact. $S$ is a semigroup with respect to the composition' of mappings.

It is not difficult to show that $S$ is closed in $H$. Thus, $S$ is compact in the topology
. of pointwise convergence on $X$. Since $X$ satisfies condition (III), for any $x \in X$, $S x=\{f x: f \in S\}$ is $G$-invariant compact convex and has a fixed point of $G$ which is a fixed point of $S$ by. Theorem 4. Therefore, by [2, Theorem 3] there exists a retraction $r \in S$ of $X$ onto $F(S)=F(G)$. Q.E.D.

If $X$ is strictly convex (i.e., for any $x, y \in X$, where $x \neq y$. and

$$
d(x, z)+d(z, y)=d(x, y)
$$

there is an $a \in(0,1)$ such that $z=W(x, y, a))$, then an analogous result holds for quasi-nonexpansive mappings. In Banach spaces similar results were given in [12].

Proposition 4. Let $X$ be a compact $W$-convex metric space which is strictly convex and satisfies condition (III). Let $G$ be a family of quasi-nonexpansive mappings on $X$ such that $G$ has common fixed points $F(G)$ in $X$. Then there exists a quasinonexpansive retraction $r$ of $X$ onto $F(G)$ for which any closed convex $G$-invariant subset of $X$ is $r$-invariant.

Proof. Let $S:=\{f: X \rightarrow X: F(f) \supset F(G)$, every $G$-invariant closed convex subset is $f$-invariant and for any $x \in X, u \in F(G), d(f x, u) \leqslant d(x, u)\}$, then $S \supset G$ and $S$ is a semigroup. Fix a point $u \in F(G)$. For each $x \in X$, put

$$
H x=\{y \in X: d(y, u) \leqslant d(x, u)\},
$$

then $H x$ is compact convex and the product topological space $H=\prod_{x \in X} H x$ is compact. Since $S$ in closed in $H, S$ is compact in the topology of pointwise convergence on $X$. By condition (III) for each $x \in X, S x$ is $G$-invariant compact convex. Hence, there is a unique element $v \in S x$ such that $d(u, v)=\min \{d(u, y): y \in S x\}$ by [8, p. 52] For any $f \in S, f v \in S x$ and $d(f v, u) \leqslant d(v, u)$, hence $f v=v$ and $v$ is a fixed point of $S$. Thus, by [2, Theorem 3] there exists a retraction $r \in S$ of $X$ onto $F(S)=F(G)$. Since $F(r)=F(G), r$ is quasi-nonexpansive. Q.E.D.

The followings are common fixed point theorems for singlevalued mappings and multivalued mappings. Let $X$ be a set, $f$ a mapping of $X$ into $X, G$ a family of mappings of $X$ into $X, T$ a mapping of $X$ into $2^{X} . f$ and $T$ are said to commute if for any $x \in X, f(T x) \subset T(f x)$. $G$ and $T$ are said to commute if every $s \in G$ and $T$ commute.
If $G$ is a semigroup acting on $X$, then $G$ and $T$ are said to commute whenever the semigroup of mappings $\{x \rightarrow s x(x \in X): s \in G\}$ and $T$ commute.

THEOREM 4. Let $X$ be a compact $W$-convex metric space satisfying condition (III), $G$ a semitopological semigroup acting on $X$. Suppose the action of $G$ on $X$ is separately continuous, nonexpansive and $\operatorname{AP}(G)$ has a left invariant mean. Let $T$ be a nonexpansive mapping of $X$ into $\mathrm{CK}(X)$. If $G$ and $T$ commute, then there exists an element $z \in X$ such that $s z=z \in T z$ for all $s \in G$.

Proof. By Proposition 3 there exists a nonexpansive retraction $r$ of $X$ onto $F(G)$ such that any $G$-invariant compact convex subset of $X$ is $r$-invariant. Define a mapping $S: X \rightarrow \mathrm{CK}(X)$ by $S x=T(r x)(x \in X)$, then $S$ is nonexpansive and has a fixed point $v \in X$ by Corollary 1 . Since $G$ and $T$ commute, $S v$ is $G$-invariant, hence $r$-invariant. In particular, $r v \in S v$. Denote $z=r v$, then we have $s z=z \in T z$ for all $s \in G$. Q.E.D.

THEOREM 5. Let $X$ be a bounded complete W-starshaped metric space satisfying condition (I), $f$ an asymptotically regular, condensing mapping of $X$ into $X, T$ a nonexpansive condensing mapping of $X$ into $K(X)$. If $f$ and $T$ commute, then there exists $a z \in X$ for which $f z=z \in T z$.

Proof. There is a fixed point $v \in X$ of $T$. Since $f$ and $T$ commute, $f^{n} v \in T\left(f^{n} v\right)$ $(n=1,2, \ldots) .\left\{f^{n} v\right\}$ is precompact because $f$ is condensing. Thus, there is a subsequence $\left\{f_{\infty}^{m} v\right\}$ of $\left\{f^{n} v\right\}$ such that $\left\{f^{m} v\right\}$ converges to some $z \in X$. Since $f$ is asymptotically regular and $T$ is upper semicontinuous, $\left\{f^{m+1} v\right\}$ converges to $z$ and $f z=z \in T z$. Q.E.D.

If $T$ is asymptotically regular, then in complete metric spaces a similar result holds. The proof can be obtained by the same method of Theorem 5 using Theorem 2 instead of Theorem 1, hence we omit the proof.

THEOREM 6. Let $X$ be a bounded complete metric space, $f$ an asymptotically regular, condensing mapping of $X$ into $X, T$ an asymptotically regular, condensing mapping of $X$ into $\mathrm{CB}(X)$. If $f$ and $T$ commute, then there exists a point $z \in X$ such that $f z=z \in T z$.

If $X$ is strictly convex, then for quasi-nonexpansive mappings and multivalued nonexpansive mappings we have a similar result to Theorem 4.

THEOREM 7. Let $X$ be a compact $W$-convex metric space which is strictly convex and satisfies condition (II). Ler $G$ be a family of quasi-nonexpansive mappings on $X$ such that $G$ has common fixed points $F(G)$ in $X$. Let $T$ be a nonexpansive mapping of $X$ into $\mathrm{CK}(X)$. If $G$ and $T$ commute, then there exists a $z \in X$ such that $s z=z \in T z$ for all $s \in G$.

Proof. Since $X$ is strictly convex, $F(G)$ is convex as in the proof of $[11$, Theorem 2]. For any $x \in F(G), s \in G, s(T x) \subset T(s x)=T x$, hence there is a fixed point of $G$ in $T x$. In fact, take a unique $u \in T x$ nearest to $x$, then $d(s u, x) \leqslant d(u, x)$ and 
$s u=u$. Define a mapping $S$ of $F(G)$ into $\mathrm{CK}(F(G))$ by $S x=F(G) \cap T x(x \in F(G))$, then for each $x, y \in F(G)$,

$$
\begin{aligned}
D(S x, S y) & =\max \left\{\sup _{v \in S x} d(v, S y), \sup _{w \in S y} d(w, S x)\right\} \\
& =\max \left\{\sup _{v \in S x} d(v, T y), \sup _{w \in S y} d(w, T x)\right\} \\
& \leqslant \max \left\{\sup _{v \in T x} d(v, T y), \sup _{w \in T y} d(w, T x)\right\} \\
& =D(T x, T y) \leqslant d(x, y) .
\end{aligned}
$$

Thus $S$ is nonexpansive and has a fixed point $z \in F(G)$ by Corollary 1. For this $z$, we have $s z=z \in T z$ for all $s \in G$. Q.E.D.

In the case that $X$ is not compact, then the following holds.

THEOREM 8. Let $X$ be a bounded complete $W$-convex metric space which is strictly convex and satisfies condition (II). Let $f$ be a nonexpansive condensing mapping of $X$ into $X, T$ a nonexpansive mapping of $X$ into $\mathrm{CK}(X)$. If $f$ and $T$ commute, then there exists an element $z \in X$ such that $f z=z \in T z$.

Proof. $F(f)$ is nonempty compact by Theorem 1 and convex because $X$ is strictly convex. Define a mapping $S$ of $F(f)$ into CK $(F(f))$ by $S x=F(f) \cap T x$ $(x \in F(f))$, then as in the proof of Theorem $7, S$ is nonexpansive and has a fixed point $z \in F(f)$ by Corollary 1. It follows that $f z=z \in T z$. Q.E.D.

\section{References}

[1] L. P. Belluce and W. A. Kirk, Nonexpansive mappings and fixed points in Banach spaces, Illinois J. Math. 11 (1967), pp. 474-479.

[2] R. E. Bruck, Jr., A common fixed point theorem for a commuting family of nonexpansive mappings, Pacific J. Math. 53 (1974), pp. 59-71.

[3] M. Furi and A. Vignoli, Fixed points for densifying mappings, Atti Accad. Naz. Lincei Rend. Cl. Sci. Fis. Mat. Natur. 47 (1969), pp. 465-467.

[4] R. D. Holmes and A. T. Lau, Nonexpansive actions of topological semigroups and fixed points, J. London Math. Soc. 5 (2) (1972), pp. 330-336.

[5] S. Itoh and W. Takahashi, Singlevalued mappings, multivalued mappings and fixed point theorems, to appear in J. Math. Anal. Appl.

[6] - Nonexpansive retractions and common fixed point theorems for singlevalued mappings and multivalued mappings, Res. Rep. Information Sci. No. A-20 (1975).

[7] A. T. Lau, Invariant means on almost periodic functions and fixed point properties, Rockey Mountain J. Math. 3 (1973), pp. 69-76.

[8] H. V. Machado, Fixed point theorems for nonexpansive mappings in metric spaces with normal structure, Ph. D. Dissertation, The University of Chicago, June, 1971.

[9] S. B. Nadler, Jr., Multi-valued contraction mappings, Pacific J. Math. 30 (1969), pp. 475-488.

[10] W. Takahashi, Fixed point theorem for amenable semigroup of nonexpansive mappings, Kōdai Math. Sem. Rep. 21 (1969), pp. 383-386.
[11] W. Takahashi, A convexity in metric space and nonexpansive mappings, I, Ködai Math. Sem. Rep. 22 (1970), pp. 142-149.

[12] - Fixed point theorems for quasi-nonexpansive mappings, Res. Rep. Information Sci. No. A-24 (1976)

DEPARTMENT OF INFORMATION SCIENCES

TOKYO INSTITUTE OF TECHNOLOGY

Oh-okayama, Meguro-ku, Tokyo, Japan

Accepté par la Rédaction le 2. 8. 1976 